\title{
Scaling of temporal correlations in densely connected networks of LIF neurons
}

\author{
Jesús Manrique ${ }^{1 *}$, Alfonso Renart ${ }^{2}$, Jaime de la Rocha ${ }^{3}$, Néstor Parga ${ }^{1}$ \\ From Twentieth Annual Computational Neuroscience Meeting: CNS*2011 \\ Stockholm, Sweden. 23-28 July 2011
}

It has been experimentally reported that neurons in cerebral cortex can fire in a decorrelated fashion despite presumably sharing a non-negligible fraction of their inputs[1,2]. Performing a scaling analysis where the synaptic strengths decrease as $1 / \operatorname{sqrt}(\mathrm{N})$, being $\mathrm{N}$ the number of neurons in the network, it has been analytically shown [1] that randomly connected networks of binary excitatory (E) ad inhibitory (I) neurons show average correlation coefficient of the activity which decreases as $1 / \mathrm{N}$ while correlations of the synaptic current components remain $\mathrm{O}(1)$. The above applies to densely connected networks in which the connectionprobability does not depend on $\mathrm{N}[1]$, in contrast to sparse networks where it is the number of connections of a neuron which is kept constant[3]. Here we present a numerical study of the dynamics of random and densely connected EI networks of current-based leaky-integrate-and-fire neurons. Preliminary results show the existence of two regimes depending on the magnitude of the external drive onto the network: one in which average correlations do not systematically decrease with $\mathrm{N}$ (regime obtained with large external currents) and one in which average correlation of voltages (Fig. 1) and spike counts decrease with $\mathrm{N}$ (regime obtained with small external current). Preliminary data indicates that the latter regime occurs in the presence of strong correlations of the current components. The lag between excitatory and inhibitory spikes also decays with the network size. These results suggest that a mechanism of active decorrelation of synaptic inputs similar to the one previously found for binary networks, also exists in networks of integrate-and-fire neurons.

\footnotetext{
* Correspondence: jesus.manrique@estudiante.uam.es

'Dpto. Física Teórica, Universidad Autónoma de Madrid, 28049,Madrid, Spain Full list of author information is available at the end of the article
}

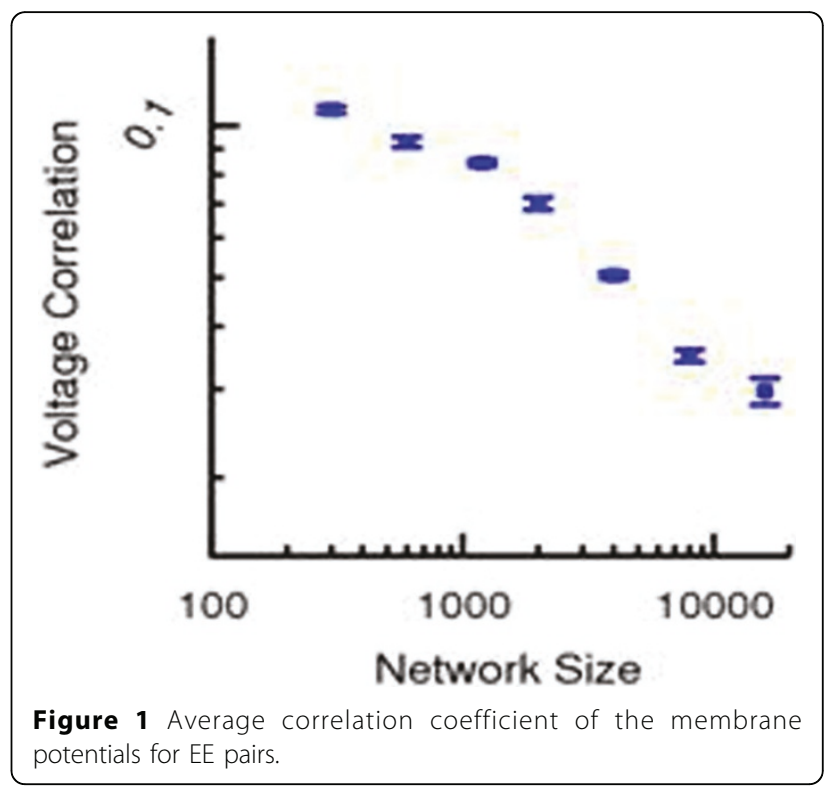

Author details

'Dpto. Física Teórica, Universidad Autónoma de Madrid, 28049,Madrid, Spain. ${ }^{2}$ Champalimaud Centre for the Unknown, 1400-038 Lisbon, Portugal. ${ }^{3}$ Institut d'Investigacions Biomèdiques August Pi i Sunyer(IDIBAPS), 08036, Barcelona, Spain.

Published: 18 July 2011

\section{References}

1. Renart A, de la Rocha J, Bartho P, Hollender L, Parga N, Reyes A, Harris K: The Asynchronous State in Cortical Circuits. Science 2010, 327:587-590.

2. Ecker A, Berens P, Keliris G, Bethge M, Logothetis N, Tolias A: Decorrelated Neuronal Firing in Cortical Microcircuits. Science 2010, 327:584-587.

3. van Vreeswijk C, Sompolinsky H: Chaos in Neural Networks with Balanced Excitatory and Inhibitory activity. Science 1996, 274:1724-1726.

doi:10.1186/1471-2202-12-S1-P249

Cite this article as: Manrique et al:: Scaling of temporal correlations in densely connected networks of LIF neurons. BMC Neuroscience 201112 (Suppl 1):P249

\section{Biomed Central}

(c) 2011 Manrique et al; licensee BioMed Central Ltd. This is an open access article distributed under the terms of the Creative Commons Attribution License (http://creativecommons.org/licenses/by/2.0), which permits unrestricted use, distribution, and reproduction in any medium, provided the original work is properly cited. 